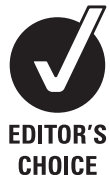

CHOICE
- An additional appendix is published online only. To view this file please visit the journal online (http://sti.bmj.com/ content/88/2.toc)

${ }^{1}$ Social and Mathematical Epidemiology Group (SAME), Department of Global Health of Development, London School of Hygiene and Tropical Medicine, London, UK

${ }^{2}$ UNAIDS, Geneva, Switzerland

\section{Correspondence to}

Sedona Sweeney, Global Health and Development, London

School of Hygiene and Tropical Medicine, WC1E 7HT, London, UK;

sedona.sweeney@Ishtm.ac.uk

This article is an abbreviated version of: http://integra.wp. tincan.co.uk/wp-content/ uploads/2012/01/UNAIDSReport.pdf

Accepted 7 November 2011 Published Online First 8 December 2011

\title{
Costs and efficiency of integrating HIV/AIDS services with other health services: a systematic review of evidence and experience
}

\author{
Sedona Sweeney, ${ }^{1}$ Carol Dayo Obure, ${ }^{1}$ Claudia B Maier, ${ }^{2}$ Robert Greener, ${ }^{2}$ \\ Karl Dehne, ${ }^{2}$ Anna Vassall ${ }^{1}$
}

\begin{abstract}
Objectives To review the literature on the potential efficiency gains of integrating HIV services with other health services.

Design Systematic literature review. Search of electronic databases, manual searching and snowball sampling. Studies that presented results on cost, efficiency or cost-effectiveness of integrated HIV services were included, focusing on low- and middleincome countries. Evidence was analysed and synthesised through a narrative approach and the quality of studies assessed.
\end{abstract}

Results Of 666 citations retrieved, 46 were included (35 peer reviewed and 11 from grey literature). A range of integrated HIV services were found to be cost-effective compared with 'do-nothing' alternatives, including HIV services integrated into sexual and reproductive health services, integrated tuberculosis/HIV services and HIV services integrated into primary healthcare. The cost of integrated HIV counselling and testing is likely to be lower than that of stand-alone counselling and testing provision; however, evidence is limited on the comparative costs of other services, particularly HIV care and treatment. There is also little known about the most efficient model of integration, the efficiency gain from integration beyond the service level and any economic benefit to HIV service users.

Conclusions In the context of increasing political commitment and previous reviews suggesting a strong public health argument for the integration of HIV services, the authors found the evidence on efficiency broadly supports further efforts to integrate HIV services. However, key evidence gaps remain, and there is an urgent need for further research in this area.

\section{INTRODUCTION}

Integration is a growing priority in the context of the AIDS response. HIV/AIDS is intrinsically linked to many other health problems. Integration has the potential to improve the quality and continuity of care for those living with HIV or bring HIV services to those who would otherwise not have access to them. ${ }^{1}{ }^{2}$ For some interventions, such as prevention of mother-to-child transmission of HIV (PMTCT) or prevention and treatment of tuberculosis (TB) co-infection, integration is clinically essential. The integration of HIV services is supported by a wide range of evidence on its clinical and public health benefit. ${ }^{3-7}$ These highlight its benefits to patients with co-morbidities, benefits in terms of continuity of care and increased access to HIV services. It has therefore been called for in a number of global policies and high-level position papers, most recently in the 2011 UN Declaration on HIV/AIDS. ${ }^{8}$ It is also commonly assumed that integration can improve programme efficiency. 2910 This is of particular interest in the current economic climate, as many countries are seeking to rationalise their health-related expenditure. However, to date, the evidence base to support this assertion remains unclear, despite the numerous reviews that focus on HIV integration more generally. $^{9-18}$ This paper therefore summarises the current evidence on the impact of integration of HIV services on the efficiency of health services, focusing on low- and middle-income countries.

Integration has been approached differently in a wide variety of settings, making the concept of integration difficult to tie down. Although there has been some consensus recently in the field of sexual and reproductive health (SRH) on terminology, ${ }^{19}$ there remain several differing discourses on integration. ${ }^{12} 20-23$ The most common understanding relates to horizontal integration or integration at the point of service delivery; this can range from structured referrals to physical incorporation providing a one-stop approach. ${ }^{21}$ Integration can also be seen as part of a wider system of co-ordination at the policy and planning, human resources and financing levels, sometimes referred as linkages. ${ }^{19}$ For the purposes of this review, we use the UNAIDS definition of programme integration: 'joining together different kinds of services or operational programmes in order to maximize outcomes, e.g. by organizing referrals from one service to another or offering one-stop comprehensive and integrated services'. W1 This includes services from a singular provider and from separate providers (within one site) where there is a clearly functional referral system.

Economic theory suggests several potential efficiency advantages at various levels of a health system arising from the integration of HIV and other health services. ${ }^{15} 24$ Integration has the potential to improve both technical efficiency (providing services or producing outputs at the lowest cost) and allocative efficiency (achieving health outcomes at a low cost). Technical efficiency focuses on using the right mix of resources to produce health services and can be assessed by measuring the unit cost of HIV services. Allocative efficiency is also concerned with whether the right 
mix of health services are provided and is commonly assessed using measures of cost-effectiveness.

Integration has the potential to improve technical efficiency through economies of scope and scale. Economies of scope, or reductions in HIV service costs from combining services, may be found through shared use of a common infrastructure, overheads and certain 'indivisible' operational resources. Economies of scale, or reductions in HIV service costs associated with increased scale of service provision, may be found where integration enables expansion of service coverage to clients who have not previously accessed them.

Beyond this, as stated above, HIV service integration may be clinically essential for the provision of cost-effective services for those with HIV (eg, the integration of HIV counselling and testing (CT) into antenatal care (ANC) services is clinically required for the provision of PMTCT). Integration can thus also contribute to an overall improvement of the allocative efficiency of HIV services. Moreover, integration may enable further efficiency gains beyond the service level. Box 1 further describes the potential effect of integration at each level of a health system, loosely based on the framework to describe integration developed by Atun et al. ${ }^{25}$

Economic evidence on integration from other sectors suggests that integration is likely to produce efficiency gains when '(1) goods/services are complementary, (2) there is a low marginal cost of added service, (3) consumers value all services and (4) there is a low correlation in demand for different services'. ${ }^{15}$ The integration of HIV and other health services potentially meets most of these requirements. First, HIV and $\mathrm{TB} / \mathrm{SRH} /$ maternal and child health services are complementary

\section{Box 1 Potential efficiency gains from integration}

1. Integration at the governance level (such as co-ordination of strategic and operation planning and performance level) may improve technical efficiency by sharing scarce resources, such as skilled planners and managers. Joint or co-ordinated planning and management, monitoring and evaluation, and reporting may also improve allocative efficiency, as (or if) planners allocate their scarce resources across interventions taking into account the relative cost-effectiveness of services.

2. At the financing level, integration may improve technical efficiency by merging the costs of separate financing systems. Co-ordinated financing systems may also reduce perverse incentives that may be created by competing programmes and thus impact allocative efficiency.

3. At the health management systems level, integration can facilitate improvements in technical efficiency through reductions in management systems costs. This can include joint procurement, sharing of middle managers, joint training and supervision, sharing of information, education and communication materials, and joint management information systems.

4. At the facility level, integration can contribute to reductions in facility costs resulting from joint utilisation of fixed factors of production.

5. For patients, integration may lead to less fragmented services, higher levels of continuity of care, better referral systems and possibly reductions in patient/community-level costs resulting from fewer visits to facilities, greater proximity of services and reduced delays in accessing treatment. services as they address co-infections, are provided at similar health services levels and may affect the same persons. ${ }^{26}$ Second, there is a potentially low marginal cost of integrated services where clinic space exists and staff already have much of the essential knowledge and skills. ${ }^{15}$ Third, HIV services may be valued by clients seeking general services, and people living with HIV may have a wide range of other unmet health needs. ${ }^{27-30}$ Fourth, there may be a low correlation in existing demand where groups of clients seeking other health services may not seek HIV-related services independently. ${ }^{15}$

\section{METHODOLOGY}

We conducted a systematic review of published and grey literature to identify studies presenting the costs and cost-effectiveness of integrated HIV services. Searches were conducted on EconLit, Global Health, Eldis and PubMed databases. Database searches were restricted to studies written in English, potentially introducing some bias to our findings. We put no date restriction on our search but found no studies published before 1990 fit our inclusion criteria. We used two different search strings to identify studies in PubMed, as listed in box W1 in the online appendix. In order to minimise the effect of publication bias, ${ }^{\mathrm{W} 2}$ we also conducted manual searches of websites of key organisations involved in HIV-related research or cost-effectiveness studies. ${ }^{i}$ We found additional references through a 'snowball sampling' process.

Inclusion criteria were designed to retain all studies, which examined the costs or efficiency consequences of integration. We focused on studies set in low- or middle-income countries as defined by the World Bank. W3 We found only one article examining integration for services targeted at populations at a higher risk of exposure to HIV; so we expanded our search to include high-income countries for this topic as we felt that it is important to include evidence on integration relevant for concentrated epidemic settings. Study inclusion/exclusion was not blinded. Cases where there was uncertainty or disagreement about the inclusion of a study were discussed between the authors and a consensus reached.

Data extraction was divided between two authors, with uniform tables filled for all included studies. We evaluated the quality of included studies using 'Drummond's checklist' to evaluate the quality of economic articles. ${ }^{\mathrm{W} 4}$ Criteria for quality analysis also included alternatives compared, whether all relevant costs were included, source of cost and effectiveness estimates and the inclusion and type of sensitivity analysis conducted. We expanded Drummond's criteria on cost measurement to include study scale. Studies determined to be of poor quality were not excluded from the review, as we wanted to present the full range of studies, including a comment on overall quality of work in this area. However, study quality was taken into account when reporting the strength of the evidence to date and interpreting our findings. Due to the complexity of integration and differences in measures, we did not attempt to quantitatively synthesise the evidence. Instead we took a narrative approach in data synthesis, as has been recommended for reviews of health systems/organisational interventions where findings are too heterogeneous for meta-analysis. ${ }^{\text {W5 }}$ This paper is adapted from a full review report that can be found on (http://www.integrainitiative.org/). In addition, a full list of references and several additional tables and

'Organisations include Abt Associates, Population Services International, Family Health International, Health and Life Sciences Partnership, Management Sciences for Health, PATH, Center for Strategic and International Studies, Population Services International, Research for Development, John Snow International, International Planned Parenthood Foundation, Population Council and Options. 
figures can be found on (http://sti.bmj.com/content/88/2.toc); these are prefixed with a ' $W$ ' in this paper.

\section{RESULTS \\ Study overview}

Database searches returned a total of 588 results. Manual searches and snowballing retrieved an additional 78 articles, totalling 666 citations in full. Fifty-one duplicates and 451 clearly irrelevant titles were excluded in the first round of title/abstract review. Reasons for exclusion included high-income setting, had nothing to do with HIV/AIDS, commentary or review paper and intervention was clearly not integrated. One hundred and sixty-four abstracts were evaluated, and full text was read and reviewed for 55 citations in total. A total of 46 studies were retained after review of the full text. A flow chart of this review selection process is contained in the online appendix (figure W1).

Of 46 studies retained, 35 were peer-reviewed and 11 were grey literature. Studies included cost analyses, cost-effectiveness analyses and least-cost analyses. Most studies included all relevant costs in their analysis, though costing methods varied. Studies either evaluated an integrated intervention against a 'donothing' comparator in which no treatment or intervention was offered or compared alternative integrated intervention strategies (such as comparative evaluations of isoniazid preventive therapy (IPT) with and without TB screening). Nine studies conducted a direct comparison of integrated and stand-alone services providing the same set of interventions. Studies used a wide variety of service output and outcome measures for cost and cost-effectiveness. For example, cost-effectiveness was measured used the cost per TB case averted, HIV infection averted and disability-adjusted life year averted. Disabilityadjusted life years are calculated as the sum of years of life lost and years of life lived in a state of less than full health. Study quality is summarised in table W1.

We divided studies into five major topic headings. First is HIV CT integrated into other health services. This includes both voluntary and provider-initiated CT for HIV, integrated into primary healthcare (PHC), SRH, home-based care, $\mathrm{MCH}$ and TB services. Second is TB services integrated into HIV care and treatment, including IPT, intensified case finding and TB treatment. These services often used $\mathrm{CT}$ as an access point for $\mathrm{TB}$ services, although several studies also included referral from any TB unit to the CT unit, indicating two-way collaboration. Third is HIV care and treatment integrated into general health services and community-based care (including palliative care, cotrimoxazole preventive therapy, PMTCT and antiretroviral therapy (ART)). The fourth examined the integration of family planning (FP) into services for HIV-positive individuals, including PMTCT and HIV care and treatment. Finally, the last topic examined HIV care and treatment and preventive outreach services integrated into other health services for populations at higher risk of HIV exposure, who may have different needs/ interactions with the healthcare system from the general public.

\section{HIV CT integrated into other health services}

We identified 17 studies which evaluated the costs and/or costeffectiveness of the integration of CT into other health services (table 1), 10 of which were peer-reviewed and seven of which were grey literature. Five of the studies ${ }^{\mathrm{W} 6-10}$ compared the cost of integrated services with those of stand-alone services, enabling an assessment of technical efficiency gains. The remaining 12 studies ${ }^{\mathrm{W} 11-22}$ provide cost estimates for different models of integrated CT or contribute to the evidence base on the cost-effectiveness of an integrated service package.
Of the five studies which present a non-integrated comparator, three were conducted in Kenya, one in Uganda and one in India. The three Kenyan studies examined introducing CT into $\mathrm{FP} / \mathrm{PHC}$ settings. The Ugandan study ${ }^{\mathrm{W} 10}$ compared different CT strategies including stand-alone, hospital-based and facilitybased home outreach. Finally, the Indian study ${ }^{\text {W8 }}$ examined the economic consequences of merging existing SRH and HIV services. All five studies found that unit costs were consistently lower for integrated services than stand-alone services, with savings between $31 \%$ and $79 \%$ of stand-alone costs. However, they were all conducted at a relatively small scale. Generally, the studies that examined CT as part of integrated service packages (ANC/PMTCT and TB/HIV) found these services to be costeffective compared with a 'do-nothing' alternative. In addition, one study ${ }^{\mathrm{W} 22}$ reported significant potential future cost savings in ART and PMTCT services from integrating CT in ANC compared with a do-nothing alternative.

\section{TB services integrated into HIV care}

We found two models ${ }^{\mathrm{W} 23} \mathrm{~W} 24$ and seven empirically based studies (some involving limited modelling) ${ }^{\mathrm{W} 17}$ W19 W25-29 examining the costs and/or cost-effectiveness of integrating TB and HIV services (table 2). All nine studies were peer-reviewed. Studies largely evaluated elements of the ' 3 I's' strategy, an integrated approach to reduce the burden of TB on HIV-positive clients through intensified TB case finding, IPT and infection control for TB, as recommended by WHO (2008). W30 Intervention elements included IPT (in some cases targeted with a tuberculin skin test), intensified case finding and cotrimoxazole preventive therapy, integrated with CT or HIV care and treatment services. CT was commonly used as an access point for $\mathrm{TB}$ services, although in some cases (eg, ProTEST), the collaboration was bi-directional (including referral from TB to HIV and vice versa). None of the studies compared a stand-alone versus integrated option, as integration is clinically required to deliver the full range of TB/HIV services to co-infected patients. Five of the seven empirical studies focused on establishing the incremental cost-effectiveness of integrated TB/HIV services compared with 'do nothing'. All studies were set in the context of generalised epidemics in sub-Saharan Africa, with the exception of one from Cambodia. ${ }^{\text {W29 }}$

The majority of studies found that integrated TB/HIV care was highly cost-effective. Five studies included the estimated TB treatment cost savings from preventive therapy; three of these found the intervention to be cost saving. None of the studies presented a before and after evaluation of changes in average costs of services as a consequence of integration or examined different models of integration, so no conclusions on technical efficiency can be drawn.

HIV care and treatment integrated into general health services We found 18 studies ${ }^{\mathrm{W} 16} \mathrm{~W} 17 \mathrm{~W} 21 \mathrm{~W} 31-45$ evaluating the costs and/ or cost-effectiveness of HIV care and treatment integrated into general health services, community-based care or PHC (table 3). Sixteen studies were peer-reviewed and two were grey literature. Only one study (Rosen et al) ${ }^{\text {W39 }}$ considered a non-integrated alternative. Of the remaining studies, 11 specified the model of integrated service delivery in use and six specified provision in conjunction with PHC but gave few details of service delivery organisation.

All of the 11 studies that specify integrated service delivery examine HIV care and treatment in a one-stop-shop setting, except Kitajima et al ${ }^{\mathrm{W} 38}$ (which evaluated referral to different providers within the same facility). Generally, the studies were 


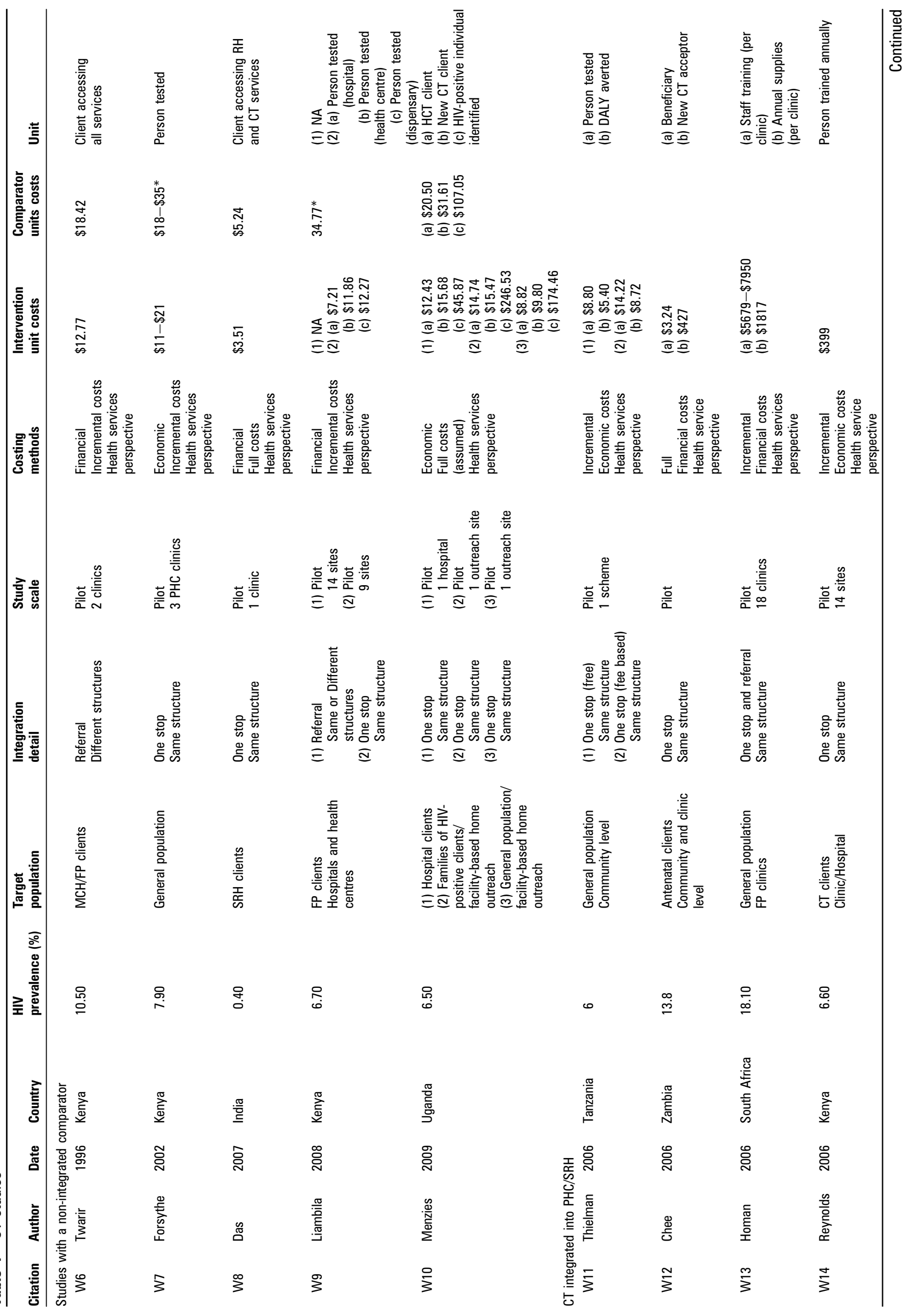




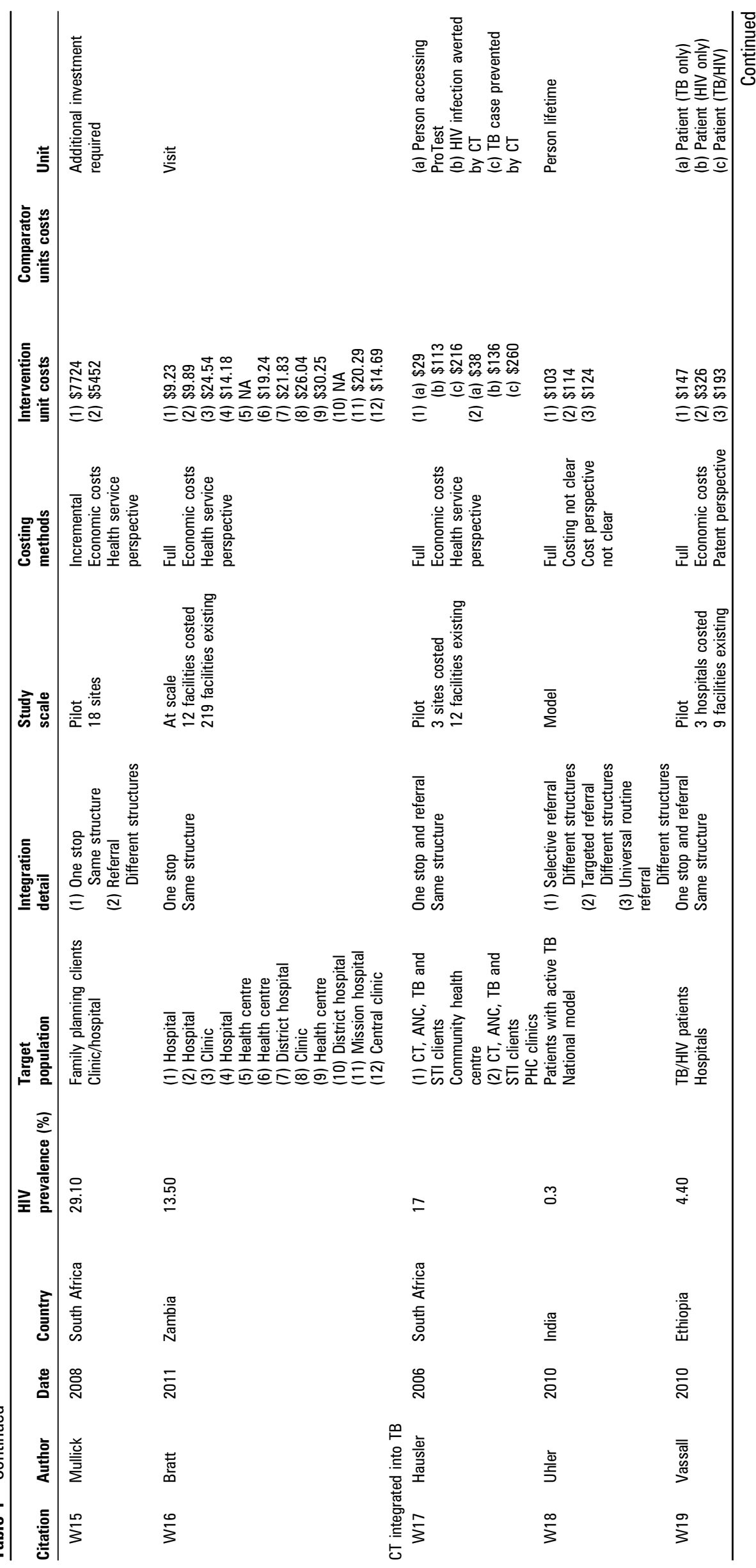




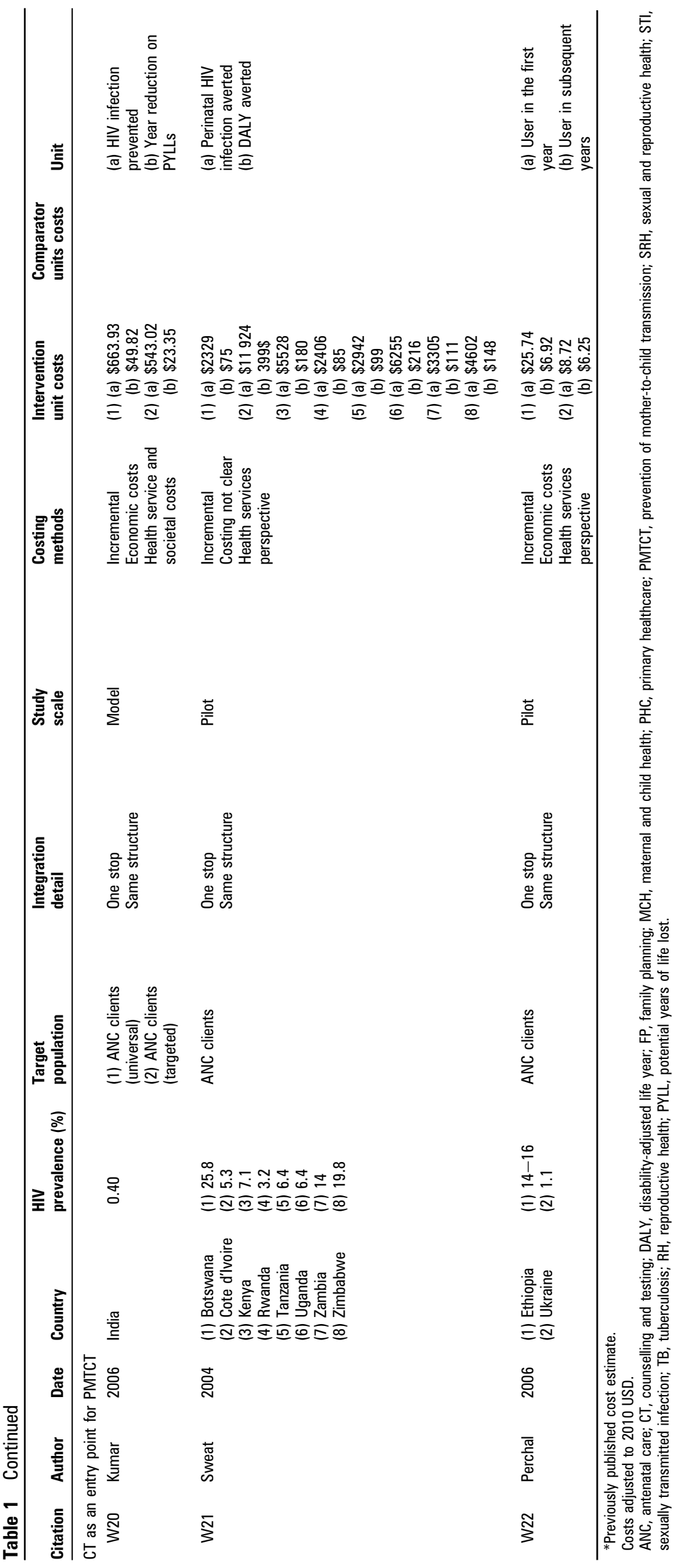




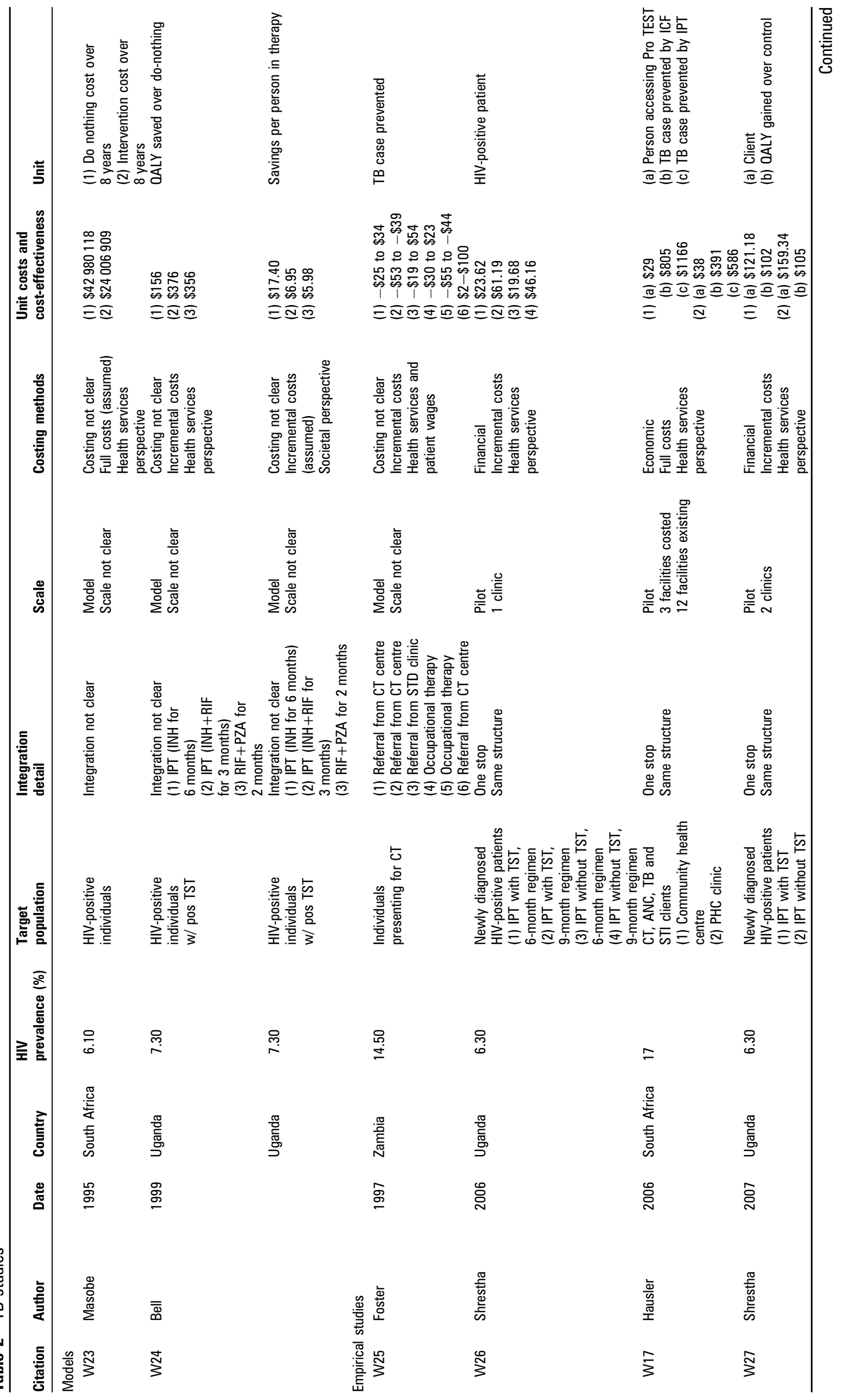




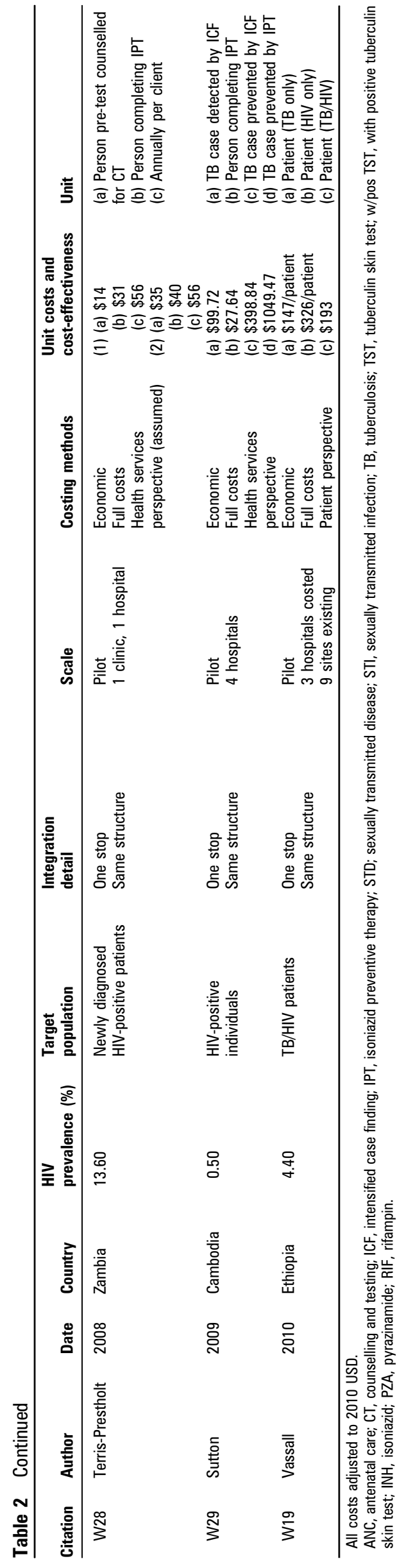

set in the context of generalised epidemics in sub-Saharan Africa, although two studies consider concentrated epidemics: one in Mexico $^{\mathrm{W} 42}$ and one in Thailand. ${ }^{\mathrm{W} 38}$ The majority of studies conclude that integrated HIV treatment and care services are feasible and cost-effective.

The one comparative study by Rosen et al ${ }^{\text {W39 }}$ found that stand-alone sites were more expensive per patient treated than some integrated models (hospital and general practitioner based) but less expensive than an integrated PHC model. When costs per patient in care and 'responding to treatment' were compared, the results for the stand-alone and integrated PHC models were equivocal and the general practitioner model was found to be more expensive. However, as this study includes only four sites (each with differing methods, financing sources and settings), no firm conclusions on whether integration or stand-alone provision of HIV care and treatment is more efficient can be drawn.

\section{FP for HIV-positive individuals}

Four studies estimated the potential cost savings from provision of FP to HIV-positive individuals through integrating FP services within PMTCT ${ }^{\mathrm{W} 46-48}$ or HIV care and treatment ${ }^{\mathrm{W} 49}$ programmes, two of which were peer-reviewed (table 4). All studies modelled costs at the national level in the context of generalised epidemics; we found no studies that empirically evaluated models of integrating FP with ART services or comprehensive care centres, despite the widespread existence of such programmes.

All studies found the provision of FP to HIV-positive individuals to be highly cost-effective or cost saving. Most studies estimate substantial savings through prevention of the costs incurred from PMTCT interventions, treatment of perinatal HIV infections, care and support for orphans and vulnerable children, and maternal deaths. No studies compared the unit costs of integrated versus stand-alone FP or HIV care and treatment services or examined the comparative costs of different models of integration.

\section{HIV services integrated with other health services for populations at a higher risk of HIV exposure}

We found three studies evaluating the integration of HIV services into other health services for key populations at higher risk of HIV exposure ${ }^{\mathrm{W} 50-52}$ (table 5). All three studies were peerreviewed. Studies examined the impact of integration on services targeted towards female sex workers, people who inject drugs, and those with mental health disorders. Two studies, set in the USA, ${ }^{\text {W5 }}$ W51 evaluated integration of mental health and/or substance abuse services into HIV care and treatment, while one set in Tanzania ${ }^{\mathrm{W} 52}$ evaluated four different approaches to provision of $\mathrm{HIV} /$ sexually transmitted disease prevention services for sex workers at truck stops. All three studies were conducted on a small scale. Two studies ${ }^{\mathrm{W} 51} \mathrm{~W} 52$ report lower unit costs per patient for integrated services, although in one, the difference was insignificant. The third study found that costs for integrated care were higher at the majority of sites and often not reimbursable by a third-party payer.

\section{DISCUSSION}

Our results show that a wide range of integrated services are cost-effective and that integration may reduce the costs of delivering some key HIV services. The best quality economic evidence focuses on the cost-effectiveness of HIV services, which require integration from a clinical perspective. This includes offering HIV CT in ANC services as part of the provision of PMTCT, providing TB services to HIV-positive clients and HIV 


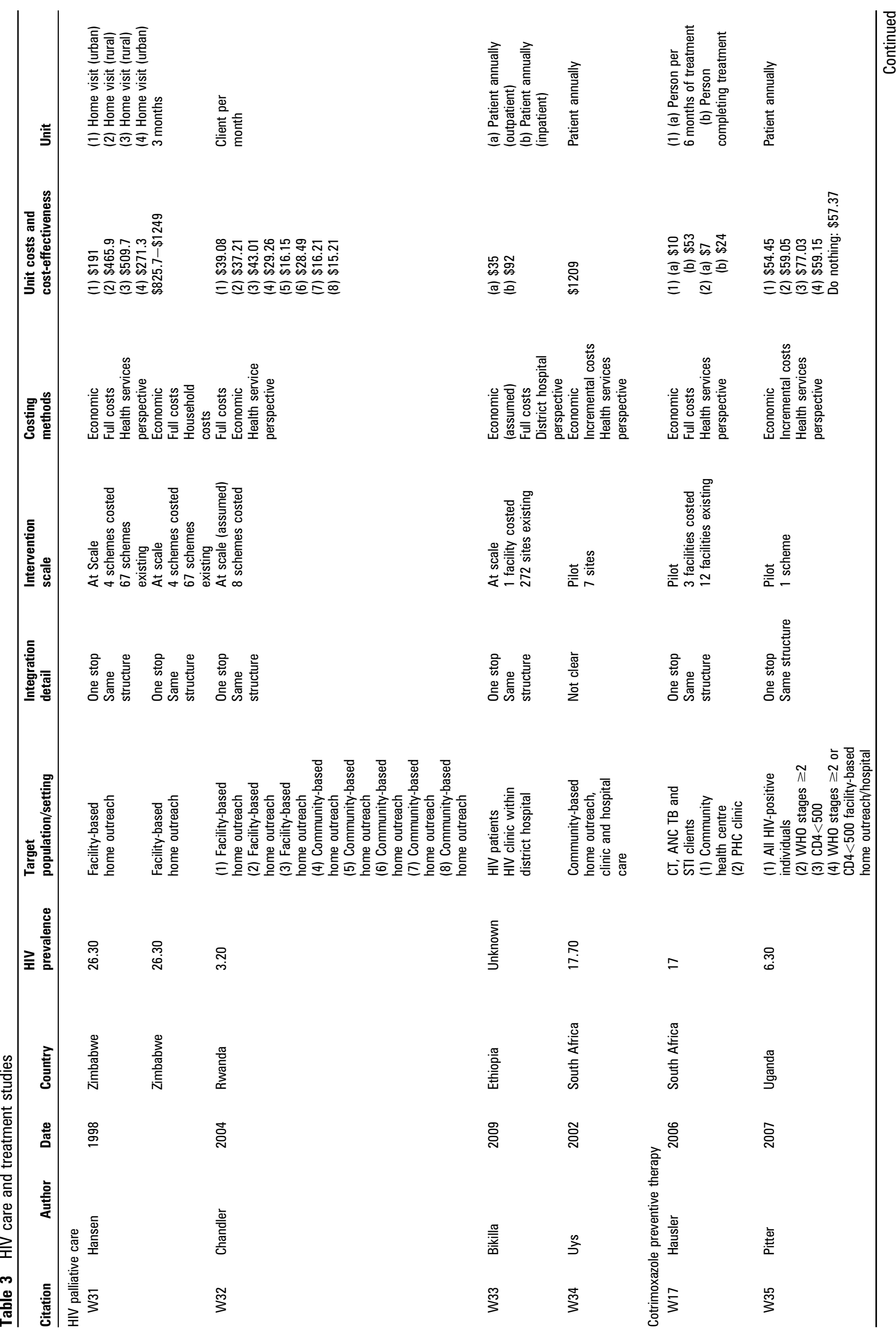




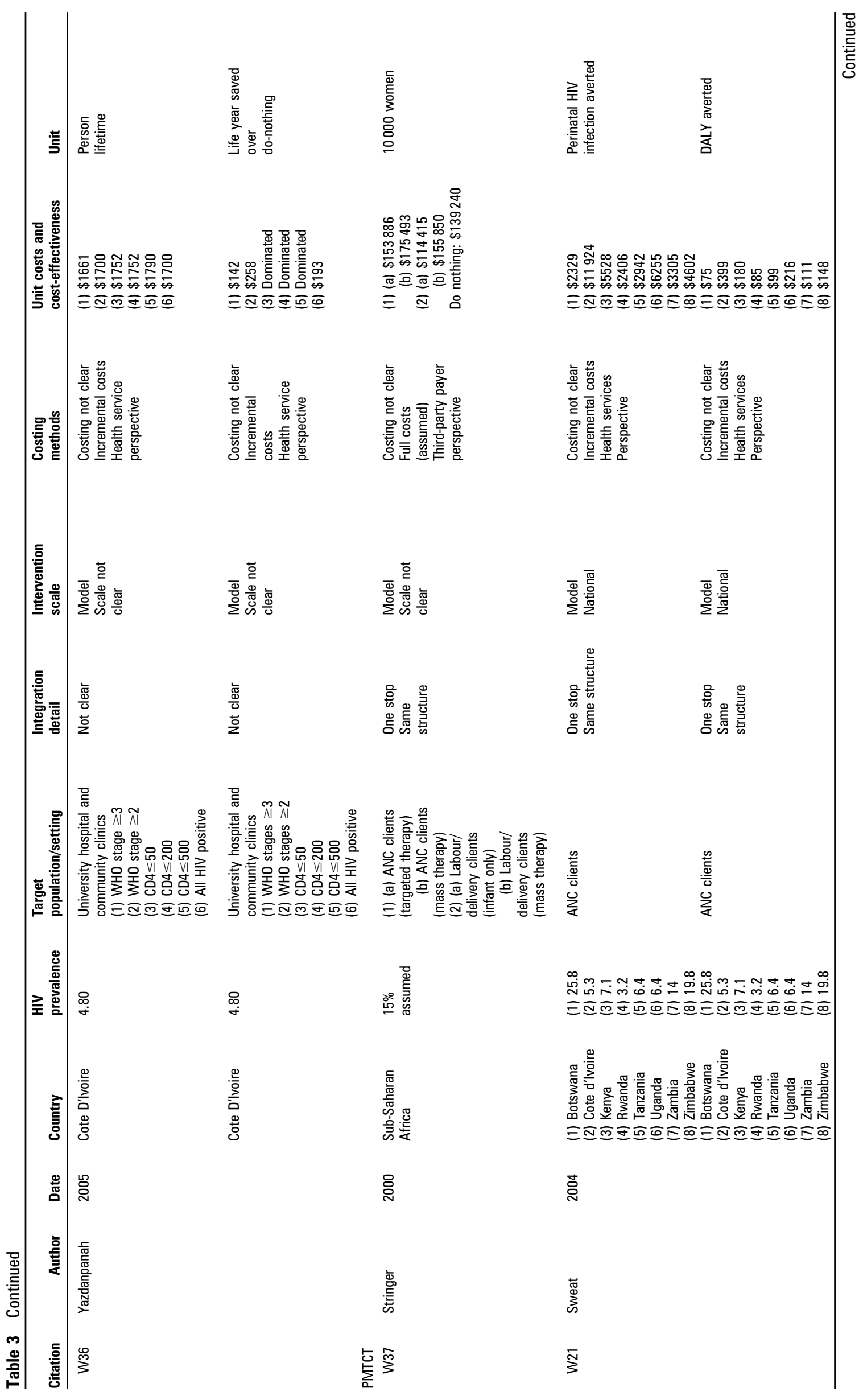




\section{National and international issues}

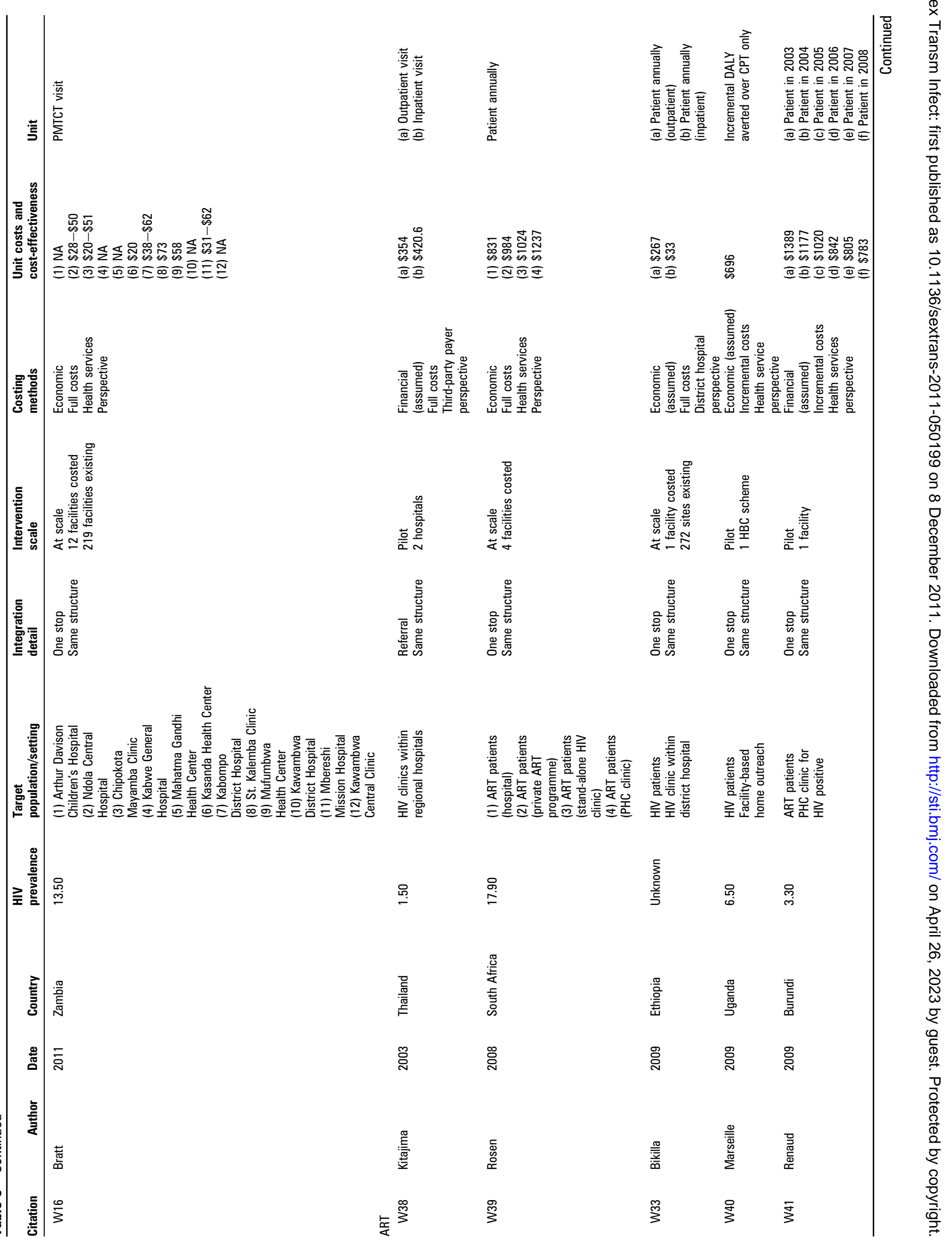




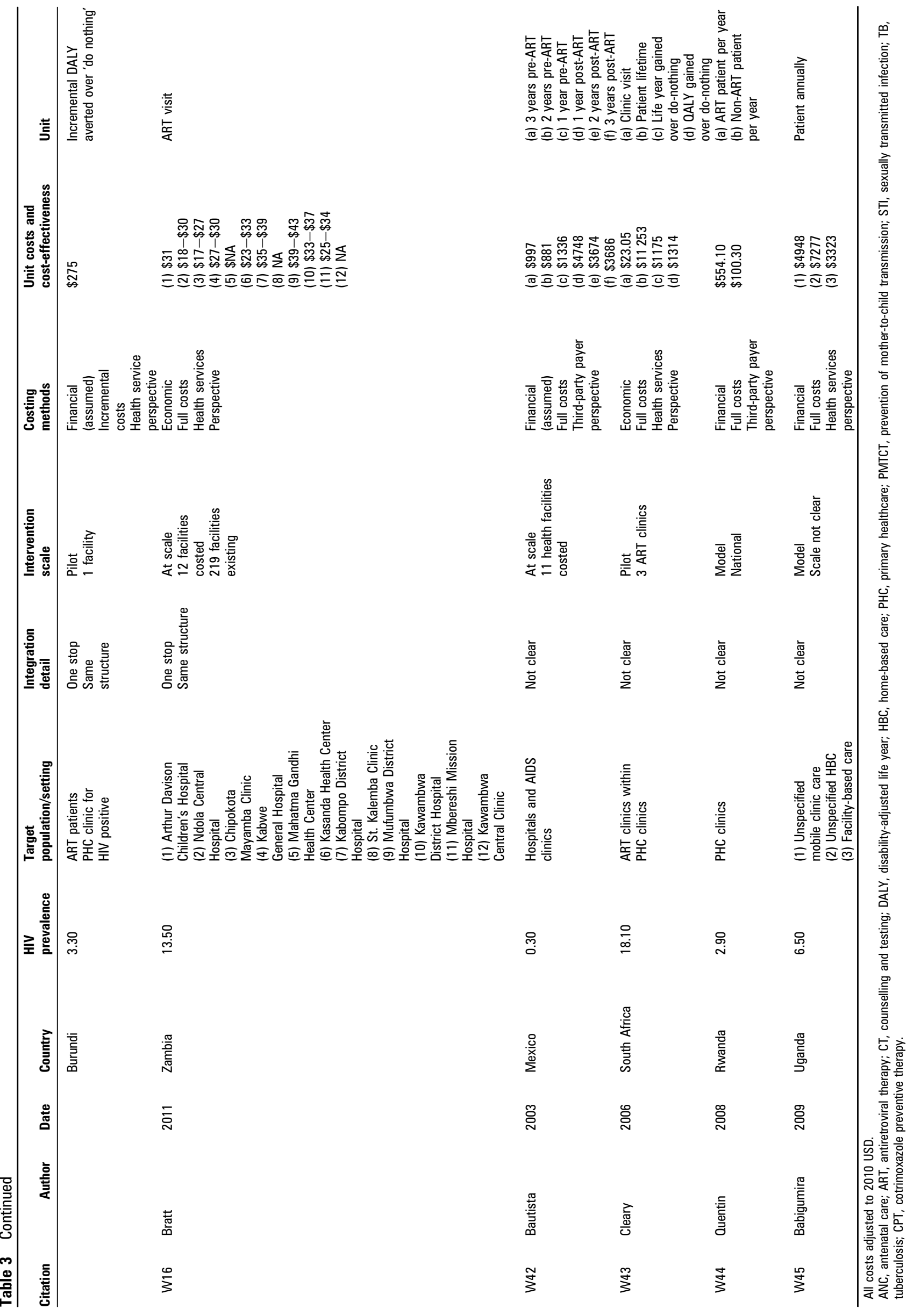


Table 4 FP studies

\begin{tabular}{|c|c|c|c|c|c|c|c|c|}
\hline Citation & Author & Date & Country & HIV prevalence & Integration detail & Costing methods & $\begin{array}{l}\text { Cost savings and } \\
\text { cost-effectiveness }\end{array}$ & Unit \\
\hline W46 & Halperin & 2009 & $\begin{array}{l}\text { (1) } 14 \text { high-prevalence } \\
\text { countries (subset) } \\
\text { (2) } 139 \text { countries }\end{array}$ & Varies & $\begin{array}{l}\text { National model } \\
\text { Service details } \\
\text { not clear }\end{array}$ & $\begin{array}{l}\text { Costing methods } \\
\text { not clear } \\
\text { Health services } \\
\text { perspective }\end{array}$ & $\begin{array}{l}\text { (1) (a) } \$ 562 \\
\text { (b) } \$ 371 \\
\text { (c) } \$ 63 \\
\text { (2) (a) } \$ 714 \\
\text { (b) } \$ 384 \\
\text { (c) } \$ 65\end{array}$ & $\begin{array}{l}\text { (a) Infant infection averted by } \\
\text { perinatal prevention } \\
\text { (b) Infant infection averted by } \\
\text { pregnancy averted } \\
\text { (c) Unintended pregnancy } \\
\text { prevented to HIV positive }\end{array}$ \\
\hline W47 & Reynolds & 2008 & $\begin{array}{l}\text { (1) Botswana } \\
\text { (2) Mozambique } \\
\text { (3) Namibia } \\
\text { (4) South Africa } \\
\text { (5) Zambia } \\
\text { (6) Ethiopia } \\
\text { (7) Kenya } \\
\text { (8) Rwanda } \\
\text { (9) Tanzania } \\
\text { (10) Uganda } \\
\text { (11) Cote d'Ivorie } \\
\text { (12) Nigeria } \\
\text { (13) Guyana } \\
\text { (14) Haiti } \\
\text { (15) Vietnam }\end{array}$ & $\begin{array}{l}\text { (1) } 24.1 \\
\text { (2) } 16.1 \\
\text { (3) } 19.6 \\
\text { (4) } 18.8 \\
\text { (5) } 17.0 \\
\text { (6) } 1.4 \\
\text { (7) } 6.1 \\
\text { (8) } 3.1 \\
\text { (9) } 6.5 \\
\text { (10) } 6.7 \\
\text { (11) } 4.7 \\
\text { (12) } 3.9 \\
\text { (13) } 2.4 \\
\text { (14) } 2.2 \\
\text { (15) } 0.5\end{array}$ & $\begin{array}{l}\text { National model } \\
\text { Service details } \\
\text { not clear }\end{array}$ & $\begin{array}{l}\text { Costing methods } \\
\text { not clear } \\
\text { Health services } \\
\text { perspective }\end{array}$ & $\begin{array}{l}\text { (1) } \$ 425695 \\
(2) \$ 1294921 \\
(3) \$ 307759 \\
(4) \$ 9036245 \\
(5) \$ 2527589 \\
(6) \$ 1984179 \\
(7) \$ 2890267 \\
(8) \$ 427605 \\
(9) \$ 1659623 \\
(10) \$ 2701425 \\
(11) \$ 592230 \\
(12) \$ 2119550 \\
(13) \text { NA } \\
(14) \$ 221298 \\
(15) \$ 76562\end{array}$ & $\begin{array}{l}\text { Saved over current practice } \\
\text { in the first year }\end{array}$ \\
\hline W48 & Stover & 2003 & 14 PEPFAR countries & Varies & $\begin{array}{l}\text { National model } \\
\text { One stop } \\
\text { Same structure }\end{array}$ & $\begin{array}{l}\text { Financial (assumed) } \\
\text { Incremental costs } \\
\text { Health services } \\
\text { perspective (assumed) }\end{array}$ & $\begin{array}{l}\text { (a) } \$ 1383 \\
\text { (b) } \$ 2767 \\
\text { (c) } \$ 702 \\
\text { (d) } \$ 383 \\
\text { (e) } \$ 138 \\
\text { (f) } \$ 2767\end{array}$ & $\begin{array}{l}\text { (a) Child HIV infection averted } \\
\text { by PMTCT only } \\
\text { (b) Child death averted by } \\
\text { PMTCT only } \\
\text { (c) Unintended HIV-positive } \\
\text { birth averted by PMTCT+FP } \\
\text { (d) Child death averted by } \\
\text { PMTCT+FP } \\
\text { (e) Unintended orphaned child } \\
\text { averted by PMTCT+FP } \\
\text { (f) Mother's life saved by } \\
\text { PMTCT+FP }\end{array}$ \\
\hline W49 & Stover & 2006 & $\begin{array}{l}\text { (1) Botswana } \\
\text { (2) Cote d'Ivorie } \\
\text { (3) Ethiopia } \\
\text { (4) Guyana } \\
\text { (5) Haiti } \\
\text { (6) Kenya } \\
\text { (7) Mozambique } \\
\text { (8) Namibia } \\
\text { (9) Nigeria } \\
\text { (10) Rwanda } \\
\text { (11) South Africa } \\
\text { (12) Tanzania } \\
\text { (13) Uganda } \\
\text { (14) Zambia }\end{array}$ & $\begin{array}{l}\text { (1) } 25.3 \\
\text { (2) } 4.4 \\
\text { (3) Unknown } \\
\text { (4) } 1.1 \\
\text { (5) } 2.1 \\
\text { (6) } 6.6 \\
\text { (7) } 11.3 \\
\text { (8) } 15 \\
\text { (9) } 3.6 \\
\text { (10) } 3 \\
\text { (11) } 18.1 \\
\text { (12) } 6 \\
\text { (13) } 6.3 \\
\text { (14) } 13.8\end{array}$ & $\begin{array}{l}\text { National model } \\
\text { Service details } \\
\text { not clear }\end{array}$ & $\begin{array}{l}\text { Costing methods } \\
\text { not clear } \\
\text { Health services } \\
\text { perspective (assumed) }\end{array}$ & $\begin{array}{l}\text { (1) } \$ 7300000 \\
\text { (2) } \$ 3400000 \\
(3) \$ 3600000 \\
\text { (4) } \$ 48000 \\
\text { (5) } \$ 4900000 \\
(6) \$ 4400000 \\
\text { (7) } \$ 2400000 \\
\text { (8) } \$ 1100000 \\
\text { (9) } \$ 3700000 \\
\text { (10) } \$ 1500000 \\
\text { (11) } \$ 14000000 \\
\text { (12) } \$ 1000000 \\
\text { (13) } \$ 18000000 \\
\text { (14) } \$ 2900000\end{array}$ & Annual net savings \\
\hline
\end{tabular}

All costs adjusted to 2010 USD.

FP, family planning; PMTCT, prevention of mother-to-child transmission.

services to those with $\mathrm{TB}$, and making FP services available to HIV-positive clients. There are number of studies that demonstrate the cost-effectiveness and cost savings of these service packages, strongly supporting further scale-up from an economic perspective. However, the cost-effectiveness of these integrated services has generally been demonstrated through models or at the pilot level, and in some areas, there is little empirical evidence to demonstrate efficiency at scale in different contexts. Additionally, none of the studies compare the relative efficiency of different models of delivery, such as one-stop versus structured referrals. None of the studies found examine whether the provision of these services may also result reductions in the unit costs of services through economies of scale or scope, and only a few examine reductions in costs to the patient. The exclusion of these factors is likely to result in an underestimation of the cost-effectiveness of these integrated services.

The integration of both CT and HIV care and treatment into general health services is feasible, and in the case of CT, the evidence consistently demonstrates that integrated CT services are likely to improve technical efficiency. It should be noted, however, that economic concerns may need to be weighed against issues such as stigma and confidentiality. Where reported, lower CT costs were primarily driven by the improved use of human resources. This suggests that the expansion of CT through existing services may place less of a burden on scarce human resources for health than expansion through stand-alone facilities (although any expansion may still require additional resourcing of staff).

Although there were several studies demonstrating the costs and feasibility of integrated HIV care and treatment, there were no high-quality studies comparing the costs of integrated services with those of stand-alone alternatives. The one study that compared costs across integrated and non-integrated sites was of an insufficient sample size. Due to the variety in services examined and methods used to measure costs, it was also hard to examine whether the costs of integrated HIV care and treatment are higher or lower than those found in other studies costing stand-alone services. Therefore, at present, no firm conclusions can be drawn on the economic benefits of integrating HIV care and treatment. 


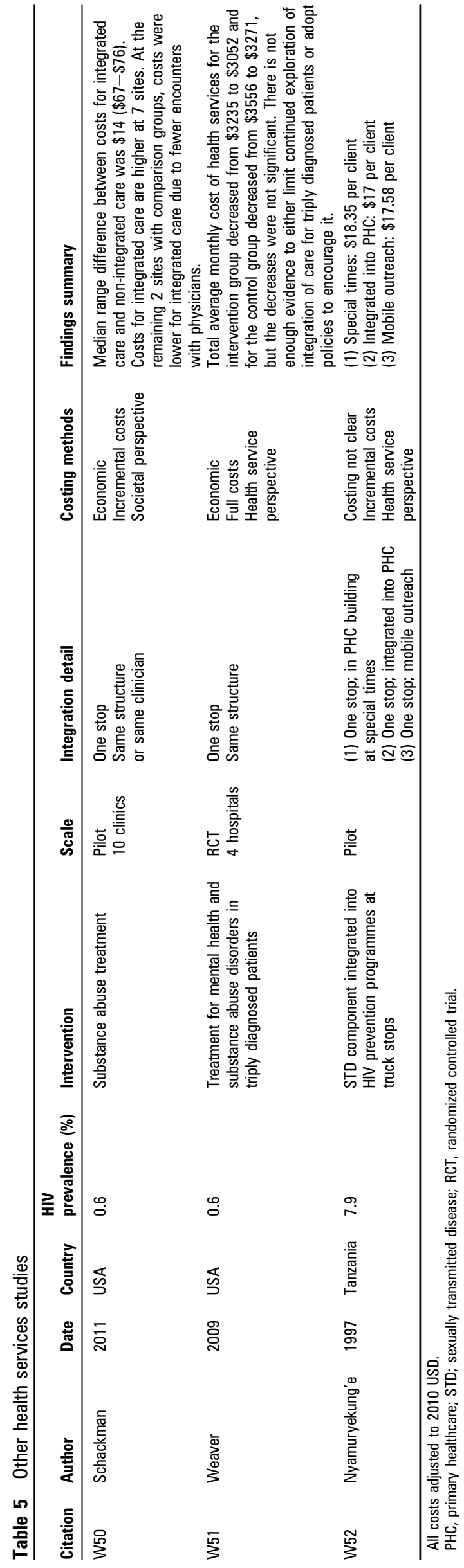

Broadly, we found that the evidence base is weak on assessing any technical efficiency gains from integration, assessing any efficiency impact beyond the service delivery level and assessing any gains from the patient perspective (box 1). Furthermore, the evidence found provides little insight into the most efficient model to employ in different contexts. None of the studies found used traditional econometric methods to estimate economies of scale or scope; where other methods were used, such as before and after comparisons, study quality tended to be poor. This is likely to reflect the fact that these methods require large sample sizes, and there is a scarcity of HIV service cost data from low- and middle-income countries. The application of data envelopment analysis, a non-parametric technique for evaluating efficiency, is beginning to be used in other sectors and could provide a solution where sample sizes are small. Even where broad analytical techniques are available, integration is complex and challenging to represent in quantitative terms. It can be best described as a continuum, progressing from linkages and service co-ordination to full integration of resources and services. ${ }^{21} 25$ New techniques, such as developing indices for integration, may provide a way forward. A recent study ${ }^{21}$ developed a scale of integration to enable cross-country comparisons of performance. Likewise, work is in progress on the Integra Initiative $e^{\text {ii }}$ to develop an index of integration. However, to date, no studies have yet explored how these scales and indices relate to cost.

This review paper has several limitations. This review only includes accessible published and grey literature and is not necessarily an accurate representation of the full range of cost data available from HIV programmes. Moreover, we were not able to account for the potential impact of political or economic context on study findings. Finally, there was a lack of consistency in cost and efficiency measures (and description of methods) across different studies, again limiting the extent that we were able to interpret and validate study results.

Our results broadly support current recommendations in international policy for integration in HIV services. Studies evaluating WHO collaborative TB/HIV treatment guidelines and 'Three I's' strategy ${ }^{\mathrm{W} 30}$ to reduce the impact of TB on those living with HIV found these interventions to be cost-effective and potentially cost saving. Studies evaluating integration of CT largely support the current WHO policy to expand providerinitiated CT for HIV in PHC settings. ${ }^{\text {W53 }}$ Finally, although there is little empirical evidence available, strong modelling evidence indicates that the steps already taken by a number of countries to integrate FP into services for HIV-positive individuals are likely to be economically beneficial.

Given the significant policy interest in scaling up integration, research on how to implement and expand integrated services is now required. In particular, there is an urgent need to assess the potential for efficiency gains from integrating HIV treatment and care into general health services, requiring large-scale costing studies. There is also almost nothing known about the impact of integration on service provision and efficiency for populations at higher risk of exposure to HIV, a key concern in concentrated epidemic settings. Moreover, for areas where cost-effectiveness is established, we recommend further operational and countrylevel research to support programme and facility managers. This should focus on identifying which models of integration are most appropriate in different settings, balancing efficiency with other service delivery objectives. Researchers should be

ii A project researching HIV/SRH integration funded by the Bill and Melinda Gates Foundation, implemented by the International Planned Parenthood Foundation, London School of Hygiene and Tropical Medicine and Population Council. 


\section{Key messages}

- The evidence to date largely supports current global policy and further efforts to integrate.

- A number of integrated HIV services have been shown to be cost-effective, though little is known about the comparative efficiency of differing integration models.

- Evidence gaps remain on economic impact of integration for HIV care and treatment and services for populations at higher risk of HIV exposure.

- Further research is necessary to identify efficiency gains from integration beyond the service level and economic gains to HIV service users.

challenged not just to assess the extent of gains to be made but also investigate the implications of different models in different settings. Research is also required on the most effective ways for programme managers to implement and promote integration at the facility level. Unfortunately, there is currently insufficient evidence to draw any lessons on the most efficient process of integration, although there are some promising examples of research in this area. ${ }^{\text {W28 }}$ Finally, none of the studies reviewed included efficiency gains at the systems and few at the patient level. The potential in this area may be significant (box 1); it is therefore highly recommended that future studies include these aspects in their assessment of integration.

In summary, given the existing evidence that largely supports HIV integration from a public health and clinical perspective, the findings of this review support further efforts to integrate. However, significant evidence gaps remain. Unfortunately, few of the studies found adequately address the central questions currently concerning many programme managers at this moment in time: not whether to integrate, but when to, how to and which model is most efficient in which setting? Investment in countrybased operational research and larger costing studies across a variety of settings is therefore urgently required to support future development of policy and programming in this area.

\section{Funding UNAIDS}

Disclaimer This research was funded by UNAIDS. The views expressed herein are those of the authors and do not necessarily reflect the official 16 policy or position of UNAIDS.

\section{Competing interests None.}

Contributors All authors were involved in aspects of study design and the analysis and interpretation of data used in the manuscript. SS and AV wrote the first draft of the manuscript. All authors contributed to subsequent drafts of the manuscript and reviewed the final version prior to submission.

Provenance and peer review Commissioned; externally peer reviewed.

\section{REFERENCES}

1. Sylla L, Bruce RD, Kamarulzaman A, et al. Integration and co-location of HIV/AIDS, tuberculosis and drug treatment services. Int J Drug Policy 2007;18:306-12.

2. World Bank. HIVIAIDS and Sexual and Reproductive Health Linkages. 2009. http:// siteresources. worldbank.org/INTPHAAG/Resources/AAGHIVAIDSSRHLinkages409.pdf

3. UNAIDS, UNFPA, FHI. New York Call to Commitment: Linking HIVIAIDS and Sexual and Reproductive Health. 2004. http://www.unfpa.org/upload/lib_pub_file/ 321 filename New\%20York\%20Call\%20to\%20Commitment.pdf
4. WHO, UNFPA. The Glion Call to Action. 2004. http://www.unfpa.org/upload/ lib_pub_file/333_filename_glion_cal_to_action.pdf

5. UNAIDS. Intensifying HIV Prevention: A UNAIDS Policy Position Paper. 2005. http:// data.unaids.org/publications/irc-pub06/jc1165-intensif hiv-newstyle en.pdf

6. The African Union Commission. The Maputo Plan of Action. Special Session of the African Union Conference of Ministers of Health. 2006. http://www.unfpa.org/africa/ newdocs/maputo_eng.pdf

7. UNAIDS. Joint Action for Results UNAIDS Outcome Framework. 2011. http://data. unaids.org/pub/BaseDocument/2010/jc1713 joint action en.pdf

8. United Nations General Assembly. Resolution $65 / 27 \overline{7}$ [Political Declaration on HIV/AIDS: Intensifying our Efforts to Eliminate HIV/AIDS]. In: Sixty-fitth Session. 2011. http://www.unaids.org/en/media/unaids/contentassets/documents/document/2011/ 06/20110610_UN_A-RES-65-277_en.pdf

9. Church K, Mayhew SH. Integration of STI and HIV prevention, care, and treatment into family planning services: a review of the literature. Stud Fam Plann 2009:40:171-86.

10. World Health Organization, UNAIDS, United Nations Population Fund, et al. Sexual \& Reproductive Health and HIV Linkages: Evidence Review and Recommendations. Geneva: International Planned Parenthood Federation, United Nations Population Fund, UNAIDS, University of California San Francisco, 2009.

11. Soto TA, Bell J, Pillen MB; HIV/AIDS Treatment Adherence, Health Outcomes and Cost Study Group. Literature on integrated HIV care: a review. AIDS Care 2004;16 (Suppl 1):S43-55.

12. Briggs $\mathbf{C} \mathbf{J}$, Garner P. Strategies for integrating primary health services in middle- and low-income countries at the point of delivery. Cochrane Database Syst Rev 2006;(2): CD003318.

13. French RS, Coope CM, Graham A, et al. One stop shop versus collaborative integration: what is the best way of delivering sexual health services? Sex Transm Infect 2006;82:202-6

14. PATH. Convergence of HIV and SRH Services in India: Impacts on and Implications for Key Populations A Literature Review. New Delhi, India, 2007. http://www.path. org/publications/detail.php?i=1503

15. Ickovics JR. "Bundling" HIV prevention: integrating services to promote synergistic gain. Prev Med 2008;46:222-5.

16. Atun $\mathbf{R}$, de Jongh T, Secci F, et al. A systematic review of the evidence on integration of targeted health interventions into health systems. Health Policy Plan 2010;25:1-14.

17. Kennedy CE, Spaulding AB, Brickley DB, et al. Linking sexual and reproductive health and HIV interventions: a systematic review. J Int AIDS Soc 2010;13:26.

18. Tudor Car L, Van-Velthoven MH, Brusamento S, et al. Integrating prevention of mother-to-child HIV transmission (PMTCT) programmes with other health services for preventing HIV infection and improving HIV outcomes in developing countries. Cochrane Database Syst Rev 2011;6:CD008741.

19. IAWG for SRH \& HIV Linkages. SRH \& HIV Linkages Resource Pack. 2010. http:// www.srhhivlinkages.org/en/srh and hiv linkages.html

20. Unger JP, DePaepe P, Greens A. A code of best practice for disease control programmes to avoid damaging health care services in developing countries. Int $\mathrm{J}$ Health Plann Manage 2003;18(Suppl 1):S27-39.

21. Shigayeva A, Atun R, McKee M, et al. Health systems, communicable diseases and integration. Health Policy Plan 2010;25(Suppl 1):i4-20.

22. Atun R, Bennett DS, Duran A. When Do Vertical (Stand-Alone) Programmes Have A Place in Health Systems? Copenhagen, Denmark: World Health Organization Regional Office for Europe, on behalf of the European Observatory on Health Systems and Policies, 2008.

23. Leichsenring K. Developing integrated health and social care services for older persons in Europe. Int J Integr Care 2004;4:e10.

24. McPake B, Kumaranayake L, Normand CEM. Health Economics: An International Perspective. London: Routledge, 2002.

25. Atun R, de Jongh T, Secci F, et al. Integration of targeted health interventions into health systems: a conceptual framework for analysis. Health Policy Plan 2010;25:104-11.

26. Lush L, Walt $\mathrm{G}$, Cleland $\mathrm{J}$, et al. The role of $\mathrm{MCH}$ and family planning services in HIV/ STD control: is integration the answer? Afr J Reprod Health 2001;5:29-46.

27. deBruyn M. HIVIAIDS and Reproductive Health: Sensitive and Neglected Issues. A review of The Literature. Recommendations For Action. Chapel Hill: IPAS, 2005.

28. Delvaux T, Nostlinger C. Reproductive choice for women and men living with HIV contraception, abortion and fertility. Reprod Health Matters 2007;15 (29 Suppl):46-66.

29. Myer L, Rebe K, Morroni C. Missed opportunities to address reproductive health care needs among HIV-infected women in antiretroviral therapy programmes. Trop Med Int Health 2007:12:1484-9.

30. Gruskin S, Firestone R, Maccarthy S, et al. HIV and pregnancy intentions: do services adequately respond to women's needs? Am J Public Health 2008;98:1746-50. 